\title{
The likelihood of GODs' existence: Improving the SN 1987a constraint on the size of large compact dimensions
}

\author{
Christoph Hanhart, ${ }^{\mathrm{a}, \mathrm{b}, 1}$ José A. Pons, ${ }^{\mathrm{d}, 3}$ Daniel R. Phillips, ${ }^{\text {a,c, }, 2}$ \\ Sanjay Reddy, ${ }^{\text {b,4 }}$

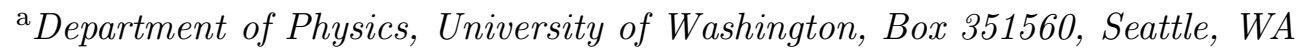 \\ 98195-1560. \\ ${ }^{\mathrm{b}}$ Institute for Nuclear Theory, University of Washington, Box 351550, Seattle, WA \\ 98195-1550. \\ ${ }^{\mathrm{c}}$ Department of Physics and Astronomy, Ohio University, Athens, OH 45701. \\ ${ }^{\mathrm{d}}$ Department of Physics and Astronomy, SUNY at Stony Brook, Stony Brook, New \\ York 11794-3800.
}

\begin{abstract}
The existence of compact dimensions which are accessible only to gravity represents an intriguing possible solution to the hierarchy problem. At present the strongest constraint on the existence of such compact Gravity-Only Dimensions (GODs) comes from SN 1987a. Here we report on the first self-consistent simulations of the early, neutrino-emitting phase of a proto-neutron star which include energy losses due to the coupling of the Kaluza-Klein modes of the graviton which arise in a world with GODs. We compare the neutrino signals from these simulations to that from SN 1987a and use a rigorous probabilistic analysis to derive improved bounds for the radii of such GODs. We find that the possibility that there are two compact extra dimensions with radii larger than $0.66 \mu \mathrm{m}$ is excluded at the $95 \%$ confidence level - as is the possibility that there are three compact extra dimensions larger than $0.8 \mathrm{~nm}$.
\end{abstract}

1 E-mail: hanhart@phys.washington.edu

2 E-mail: phillips@phy.ohiou.edu

3 E-mail: jpons@neutrino.ess.sunysb.edu

4 E-mail: reddy@phys.washington.edu 


\section{Introduction}

The possibility that there exist large, compact, space-like dimensions accessible only to gravity, but not to the standard model particles, has recently been suggested as a solution to the hierarchy problem $[1,2]$. If such compact spatial dimensions do exist then the fundamental Planck scale could be close to the electro-weak scale, with gravity becoming comparable in strength to other interactions at energies of order a few $\mathrm{TeV}$. At present, the strongest constraint on the existence of compact Gravity-Only Dimensions (GODs) such as these is derived from the SN 1987a neutrino signal. The argument, put simply, is that if too much of the available energy is radiated to the GODs then the neutrino signal seen on terrestrial detectors would have differed markedly from that actually observed. This argument allowed Arkani-Hamed et al. (ADD) to place order-of-magnitude bounds on the radii of the GODs [2]. Subsequently, an attempt to improve the analysis of Ref. [2] was made by Cullen and Perelstein in Ref. [3], who found $R<0.3 \mu \mathrm{m}(R<0.4 \mathrm{~nm})$ for 2 (3) compact extra dimensions. Further improvements to the calculation of the crucial Kaluza-Klein (KK) graviton emissivities were made in Ref. [4]. These calculations tended to confirm that the original estimate of Ref. [2] was indeed within an order of magnitude of the correct number, although they resulted in bounds a factor of two or three weaker: $R<0.71 \mu \mathrm{m}(R<0.85 \mathrm{~nm})$. However, as indicated in Ref. [3], to obtain truly accurate bounds on the size of the GODs - and, by inference, the fundamental Planck scale - one would have to incorporate the contribution to the emissivities for the graviton emission to the extra dimensions into a numerical code for PNS evolution. In contrast, Refs. [3,4] both used the simple criterion suggested by Raffelt [5], which requires only that the emissivity (energy radiated per unit mass and per unit time) of some exotic particle be less than $10^{19} \mathrm{ergs} / \mathrm{g} / \mathrm{s}$.

This bound is based on the time-scale of several seconds over which the handful of electron-type anti-neutrino events from SN 1987a were detected in Kamiokande [6] and IMB [7]. These detections confirmed the standard scenario of core-collapse Supernovae: neutron stars are born in the aftermath of successful supernova explosions, as the stellar remnant becomes gravitationally decoupled from the expanding ejecta [8-12]. The ambient conditions in a newly born neutron star, also called a proto-neutron star (PNS), are so extreme densities of order $10^{14} \mathrm{~g} / \mathrm{cm}^{3}$ and temperatures between 10 and 50 $\mathrm{MeV}$ - that the neutrino mean-free path is much smaller than the stellar radius. Consequently, the enormous gravitational binding energy gained during the stellar collapse $\left(\sim 2-3 \times 10^{53} \mathrm{ergs}\right)$ is stored inside the PNS and slowly released by neutrino diffusion on a time scale of tens of seconds. This picture meshes nicely with the long duration of SN 1987a's neutrino signal, since the neutrinos must have diffused, and not free-streamed, out of the PNS. In this context, any weakly-interacting particle which couples to nucleons and 
can freely stream out of the star easily competes with neutrinos as a means of transporting energy away from the stellar interior. Such an additional heat sink would accelerate the cooling and remove a significant fraction of the binding energy. The late-time neutrino signal, which is fueled by the heat stored in the core, is particularly sensitive to these effects, and it would be drastically altered if the couplings to exotic particles were strong enough.

It is this physics that facilitates the use of the supernova neutrino signal to place bounds on the amount of energy lost as exotic radiation. Stringent bounds have been placed on the properties of a number of novel, weaklyinteracting, light-particle species. Initially, these included limits on the neutrino magnetic moment, the mass of the axion, the strength of right-handed neutrino interactions and the sterile-neutrino production rates [13-18]. Subsequently, several authors have employed Raffelt's bound on the total emissivity due to these exotic energy-loss mechanisms - or analogous bounds on the luminosity [19] - as a simple means of enforcing the constraint provided by the SN 1987a neutrino signal. However, since the emission rates are strong functions of temperature, the bounds on exotic-particle couplings which are deduced from such arguments will depend crucially on the fiducial value of temperature at which the exotic-particle emissivity is calculated. Not surprisingly, since the temperature of the newly-born neutron star varies with both position and time, the fiducial temperature is rather loosely defined, with values between 10 and $70 \mathrm{MeV}$ appearing in the literature. Furthermore, exotic cooling mechanisms will lower the temperature inside the PNS core, and so the appropriate fiducial temperature is really a function of the emissivity of these processes. The strong temperature dependence of these emissivities ultimately means that these ambiguities can easily change the resulting bound by more than an order of magnitude.

Thus in order to establish more accurate bounds it is necessary to perform detailed simulations of the effect of exotic radiation on the neutrino signal. So far such simulations have only been carried out for the case of axion radiation $[18,20]$. These simulations tend to confirm the picture outlined in the previous two paragraphs. Therefore in this work we report on the first selfconsistent simulations of the PNS core which test the effect of GODs on the SN 1987a signal. In $\S 2$ we present a summary of the early stages of the life of a neutron star and the effect of additional energy loses. In order to make these simulations as realistic as possible we use the most recent calculations of the KK-graviton emissivity [4], which differ by roughly a factor of five from earlier, more schematic, calculations [3].

Another important drawback of former analyses is the lack of a well-established selection criteria to disregard a given model. As emphasized in Ref. [21], the sparseness of the SN 1987a data calls for a Likelihood analysis. In $\S 3$ we outline the basics of the likelihood formalism which allows for marginalization 
over uncertain parameters, as well as the derivation of a bound with a clear probabilistic interpretation and unambiguously-defined confidence levels. Our results, and a discussion of them, are presented in $\S 4$.

\section{PNS Evolution with Additional Sources of Energy Loss and SN 1987a signal}

Core-collapse supernovae are incredibly rich physical systems, which include many complicated phenomena. Indeed there is still much debate about basic features like the details of the explosion mechanism $[22,23]$. What is, however, now well-established is that the birth and subsequent evolution of the PNS located in the inner region of the supernova provides the main source of neutrinos during the first several seconds after core bounce. Nearly all of the binding energy gained during the collapse is trapped inside the PNS and this energy is radiated by neutrino diffusion over several tens of seconds [811]. These time scales and the associated neutrino luminosities are affected by several pieces of physics, including the total mass of the PNS, and both the nuclear equation of state (EOS) and neutrino opacities at supra-nuclear density. None of these quantities can be determined in a model-independent approach, but earlier studies have explored the model sensitivities by using different EOSs and the associated self-consistently-calculated opacities [11]. These studies indicate that the critical physical parameter that determines neutrino luminosity in the first several seconds is the PNS total mass. The uncertainties associated with the properties of dense hadronic matter do not affect these luminosities greatly, provided that the opacities are calculated consistently with the EOS, because important feedbacks between the EOS and the opacities tend to reduce the differences. The protoneutron star evolution code employed for this study has been described in detail in Refs. [11,12]. Details regarding the neutrino opacities employed can be found in Ref. [24]. In a recent paper the rates for the emissivities of KK-gravitons in dense matter were computed in a model independent way using low-energy theorems which relate the emissivities to the well measured nucleon-nucleon cross sections [4]. In a world with $n=2$ or $n=3$ compact GODs the KK-graviton emissivity of neutron-star matter can be fitted to within 5\% accuracy over a range of temperatures from 15 to $30 \mathrm{MeV}$ and a range of densities from $0.5 n_{0}$ to $3 n_{0}$, where $n_{0}=0.16 \mathrm{fm}^{-3}$ is the nuclear saturation density. The result, which includes contributions from $n n, p p$, and $n p$ collisions is:

$$
\frac{d E}{d t}=a_{n}\left(\frac{n_{B}}{n_{0}}\right)\left(\frac{T}{10 \mathrm{MeV}}\right)^{p_{n}} \chi\left(X_{n}, X_{p}\right) \mathrm{MeV} / \text { baryon } / \mathrm{s}
$$


with $p_{2}=5.42, a_{2}=5.1 \times 10^{4} R_{2}^{2}, p_{3}=6.5, a_{3}=1.4 \times 10^{16} R_{3}^{3}$. Here, $n_{B}$ is the baryon number density, $T$ is the temperature, and the size of the extra dimensions, $R_{n}$, is given in $\mathrm{mm}$ ?. The emissivity depends on the composition of matter through the function

$$
\chi\left(X_{n}, X_{p}\right)=X_{n}^{2}+X_{p}^{2}+4 X_{n} X_{p}\left(\frac{T}{10 \mathrm{MeV}}\right)^{-0.44},
$$

where $X_{n}$ is the neutron fraction, and $X_{p}=\left(1-X_{n}\right)$ is the proton fraction. The weak, residual, temperature dependence in the function $\chi$ arises because the $n p$ cross section decreases as approximately $1 / \sqrt{E_{c m}}$, where $E_{c m}$ is the center of mass energy of the colliding nucleons, while the $n n$ cross section is roughly independent of energy, for the energies of interest. Over the range of values of proton fraction and temperature encountered in the PNS the function $\chi$ changes by less than $10 \%$ from the case of pure neutron matter, when $\chi=1$. Thus, the total emissivity is a weak function of the proton fraction $X_{p}$, and in our simulations we assume, for simplicity, that $X_{n}=1$.

In this paper we will put limits on permitted values of the coefficients $a_{2}$ and $a_{3}$, assuming the temperature-dependencies $T^{5.42}$ and $T^{6.5}$. Thus, ultimately our results are valid for the coupling to any exotic particle which results in these temperature-dependencies. By placing limits on the coefficients $a_{n}$, we are led, in this case, to bounds on $R_{n}$ - the radii of the extra dimensions but the simulations, philosophy, and likelihood formalism discussed here can be applied in a much broader context. The count rate in the detectors is sensitive to the neutrino luminosities and spectrum. The procedure employed to obtain the count rates is described in detail in earlier work $[11,12]$. In addition, here we include the effects due to the neutron-proton mass difference on the anti-neutrino absorption cross sections and correct the fiducial mass of the IMB detector to 6 ktons [25]. Both lead to better agreement between the two detectors.

The equilibrium diffusion approximation employed in the numerical code provides a fair description of the total neutrino luminosity and its time structure. However, additional assumptions are required if an anti-neutrino spectrum is to be derived from this information. In particular, to obtain an average anti-neutrino temperature - denoted here by $T_{\bar{\nu}_{e}}$-we assume that the neutrinos leaving the protoneutron star are thermalized inside a neutrinosphere and have a Fermi-Dirac spectrum with zero chemical potential. The neutrinosphere was chosen to be at an optical depth of $2 / 3$ in accordance with the usual def-

5 In the non-degenerate limit Ref. [4] derived an analytic formula with $p_{2}=5.5$, $p_{3}=6.5$, and $a_{2}=5.6 \times 10^{4} R_{2}^{2}, a_{3}=1.5 \times 10^{16} R_{3}^{3}$. One might perhaps be justified in using these formulae, but instead we have chosen to use more accurate fits to the full result of Ref. [4]. 


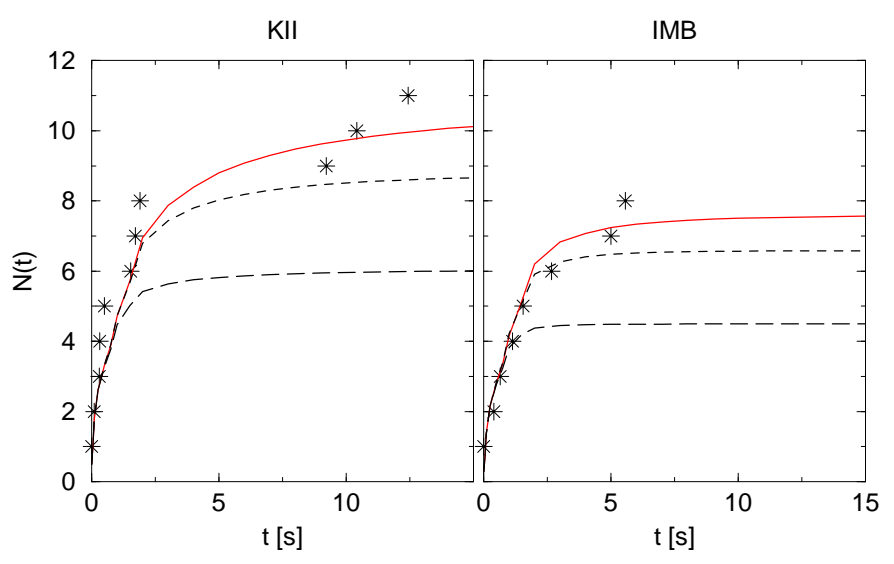

Fig. 1. Comparison of the results of our simulations for a PNS with a baryonic mass of $M=1.6 M_{\odot}$ to the data from $S N 1987 a . N(t)$ denotes the integrated number of counts. We show results from a model without graviton emission (solid line), as well as the results with graviton emission for $a_{2}=0.01 \mathrm{MeV} /$ baryon $/ \mathrm{s}$ (short-dashed line) and $a_{2}=0.1 \mathrm{MeV} /$ baryon/s (long-dashed line).

inition. However, it must be borne in mind that the neutrino mean-free paths are energy dependent, and thus the concept of a neutrinosphere is only approximate. In the next section we will study the effect of those uncertainties related to this definition of the neutrino temperature by exploring the sensitivity of the count rates and the deduced bounds to changes in our prescription. In Fig. 1 we show the number of accumulated counts as a function of time, $N(t)$, in Kamioka (left panel) and IMB (right panel). The results shown are for a baryonic mass of $1.6 \mathrm{M}_{\odot}$ (with a final gravitational mass of $1.46 \mathrm{M}_{\odot}$ ), and an anti-neutrino temperature defined as above. They include the case without KK-graviton emission, and two cases with radiation to the GODs included, namely: $a_{2}=0.01 \mathrm{MeV} /$ baryon $/ \mathrm{s}$ and $a_{2}=0.1 \mathrm{MeV} /$ baryon/s. As argued in the introduction, KK-gravitons steal energy from the core and thereby dramatically suppress the late-time neutrino emission. Note that the early-time neutrino emission is not strongly influenced by the existence of GODs because these neutrinos are emitted from the lower density and lower temperature regions of the star where the graviton emissivity is small. However, after about 2-4 seconds, neutrino losses provoke a strong compression and heating of the star $[8,10,11]$, which activates the KK-graviton emissivity. Consequently, the total number of neutrino counts, which clearly depends on the total amount of energy radiated in neutrinos, drops as $a_{2}$ is increased.

This total number of counts also has a significant dependence on the PNS mass. Indeed, the mass of the PNS is generally the most important factor in the neutrino signal [11]. However, it is worth noting that, if a sufficiently important additional sink of energy is included in the PNS simulation, the time-structure of the signal actually becomes less sensitive to the precise value of the PNS mass. This happens because more massive stars attain higher temperatures, which in turn results in enhanced graviton emission. Consequently, much of 
the additional binding energy reservoir in a more massive star is carried out by exotic particles, and the resultant neutrino luminosity is not dissimilar to that from PNSs of lower mass. This was already noticed in Ref. [20] for the axion case.

\section{From simulations to bounds: the desirability of a likelihood anal- ysis}

Nevertheless, this "mass-independence" only arises for large values of the couplings $a_{2}$ and $a_{3}$. More typically we face the problem of trying to extract bounds for these couplings from the neutrino data in the face of significant uncertainties in the PNS mass $M$ and the anti-neutrino temperature $T_{\bar{\nu}_{e}}$. Therefore a key question is how to perform a consistent analysis of the results and derive a bound on the coupling of the exotic particles. Studies similar to ours for the case of axion emission [18,20], based their investigations on either the time required to accumulate $90 \%$ of the counts $(\Delta t(90 \%))$ or the total number of counts detected $N^{\text {tot }}$.

Not surprisingly, both $N^{\text {tot }}$ and $\Delta t(90 \%)$ decrease with increasing $R_{2}$. The variation in the total number of counts is moderate and very sensitive to the variation of all three parameters studied: $a_{2}, T_{\bar{\nu}_{e}}$ and $M$. Thus, it makes more sense to study an observable that is relatively insensitive to $T_{\bar{\nu}_{e}}$ and M. $\Delta t(90 \%)$ is such a quantity. However, it is still difficult to move from the values of $\Delta t(90 \%)$ to a confidence level for a bound on $R_{2}$. One might argue that a simple criterion is to disregard those radii $R_{2}$ which lead to $\Delta t(90 \%)$ lower than one half of the value for the $a_{n}=0$ case. Using this criterion we find that:

$$
R_{2}<\left(\begin{array}{lll}
0.4 & 0.9) \mu \mathrm{m}
\end{array}\right.
$$

for PNS masses in the range $M=\left(\begin{array}{l}1.5 \ldots \\ \text {. 2.0 }\end{array}\right) M_{\odot}$. However, there is no guarantee that the result for $a_{n}=0$ is the "correct" answer. The performance of a model that badly overestimates the number of counts in the $a_{n}=0$ case might well be improved by the presence of GODs. So, if we adopt an alternative approach and disregard those values of $R_{2}$ which lead to a time scale that is less than one half of the value found in the SN 1987a data we find:

$$
R_{2}<\left(\begin{array}{lll}
0.2 \ldots & 0.4
\end{array}\right) \mu \mathrm{m}
$$

These, however, are rather arbitrary criteria. There is really no statistical 
basis [26] for judging what constitutes a successful reproduction of the experimental data, and thus no way to define confidence levels.

In light of these issues we seek an analysis tool that will allow us to average over parameters which are not well-known (such as the mass of the PNS and $T_{\bar{\nu}_{e}}$ ) and also give a clear criterion that allows us to decide what values of the parameter space are excluded by the data. To this end we now pursue a "likelihood" analysis. The goal of this analysis is the probability that the coupling $a_{n}$ is smaller than some specified value $a_{n}^{0}$, given the data, and a certain set of model assumptions. We denote this probability by $\operatorname{prob}\left(a_{n} \leq a_{n}^{0} \mid\{d a t a\}, I\right)$, where the $I$ indicates the set of physical assumptions which underly the simulations discussed in the previous section. Note that the assumptions denoted by $I$ do not include information about $M$ or $T_{\bar{\nu}_{e}}$. Our goal is to relate $\operatorname{prob}\left(a_{n} \leq a_{n}^{0} \mid\{d a t a\}, I\right)$ to a function we can calculate directly from our model. That function is the Likelihood function $\mathcal{L}_{D}\left(\{d a t a\} \mid a_{n}, M, T_{\bar{\nu}_{e}}, I\right)$, which is the the probability that the data actually taken at a detector $D$ (KII or IMB) arises given a set of model assumptions $(I)$ and certain specific values for the key quantities $M, T_{\bar{\nu}_{e}}$, and $a_{n} \square$. Assuming that the data obeys Poisson statistics, the likelihood for the neutrino signal in a single detector can then be expressed in terms of the model prediction for the count rates at the times where an event happened and the total number of events actually seen [27]

$$
\mathcal{L}_{D}\left(\{\text { data }\} \mid a_{n}, M, T_{\bar{\nu}_{e}}, I\right)=\left[\prod_{i=1}^{N^{\text {tot }}} \frac{d N_{D}\left(t_{i}^{D}\right)}{d t} \Delta t\right] e^{-N_{D}}
$$

when $N_{D}$ is the total number of observed neutrino arrivals, and $t_{1}^{D}, t_{2}^{D}, \ldots$ are the times at which neutrinos actually arrived in the detector $D . N_{D}(t)$ is the total number of neutrinos that the model with assumptions $I$, PNS mass $M$, anti-neutrino temperature $T_{\bar{\nu}_{e}}$, and exotic-coupling $a_{n}$ predicts will have arrived in the detector $D$ up until the time $t . \Delta t$ can be any interval small enough that the probability of detecting more than one count in any one bin can be taken to be negligible. Ultimately it will be absorbed into an overall normalization constant. Note that in order to get the full answer for the likelihood $\mathcal{L}$ the product of the likelihoods for KII and IMB must be taken (i.e., $\mathcal{L}=\mathcal{L}_{K I I} \times \mathcal{L}_{I M B}$ ).

The likelihood function provides us a with quantitative tool to compare models of PNS evolution. Since we treat not only the coupling to extra dimensions, $a_{n}$, but also $M$ and $T_{\bar{\nu}_{e}}$, as parameters, the likelihood function is a function in

6 We could, of course, have allowed for explicit variation of other parameters of the PNS simulation, and not simply included them in $I$, as we do when we calculate $\mathcal{L}_{D}\left(\{d a t a\} \mid a_{n}, M, T_{\bar{\nu}_{e}}, I\right)$. The formalism can easily be extended in this way if it becomes clear that some other physical parameter affects the neutrino signal strongly. 
a three-dimensional space. This function has a minimum at a baryonic mass of $M=1.5 M_{\odot}, a_{2}=0$ and $T_{\bar{\nu}_{e}}=1.1 T_{\nu}^{o} . T_{\nu}^{o}$ denotes the "reference anti-neutrino temperature" defined above using the optical-depth prescription. We can now assess all other models by looking at the log of the ratio of their likelihood of a particular model to this "most likely" model:

$$
q\left(a_{n}, M, T_{\overline{\nu_{e}}}\right)=-\log \left(\frac{\mathcal{L}\left(\{\text { dat } a\} \mid a_{n}, M, T_{\bar{\nu}_{e}}, I\right)}{\mathcal{L}\left(\{\text { data }\} \mid 0,1.5 M_{\odot}, 1.1 T_{\nu}^{o}, I\right)}\right)
$$

The function $q$ is then a function in this same three-dimensional space.

For the case of two GODs we discuss first the situation where these dimensions have zero radius, and so $a_{2}=0$. Contours of $q$ in the resulting twodimensional $x-M$ plane are shown in the left panel of Fig. (2). Here the horizontal axis is the temperature normalized to the reference anti-neutrino temperature, $x=T_{\bar{\nu}_{e}} / T_{\nu}^{o}$, which is varied to explore the sensitivity of the results to deviations of the neutrino temperature from that obtained using the optical depth prescription. We see that although $M=1.5 M_{\odot}, T=1.1 T_{\nu}^{o}$ is indeed a minimum of the function $q$ it is a rather shallow minimum: varying the neutron-star mass and the anti-neutrino temperature over the range considered here does not reduce the likelihood greatly. Ultimately this reflects the weakness of the constraint that the SN 1987a data provides for these parameters.

The situation is rather different as we move away from $a_{2}=0$. In the middle panel we display similar likelihood contours in the $M-\log \left(a_{2}\right)$ plane for the case $T_{\bar{\nu}_{e}}=T_{\nu}^{o}$, while the right panel shows contours in the $x-\log \left(a_{2}\right)$ plane for the baryonic mass $M=1.6 M_{\odot}$. (The pattern of contours shown is typical, and does not change significantly if different values of $T_{\bar{\nu}_{e}}$ and $M$ are chosen.) These panels show that the likelihood function decreases rapidly for $a_{2} \geq 10^{-2}$-regardless of the values of the poorly-known parameters $M$ and $T_{\bar{\nu}_{e}}$. Such large values of $a_{2}$ are essentially two orders of magnitude less likely than the "most likely" model. This is a contrast to smaller values of $a_{2}$, where the differences in likelihood are comparable to those seen in the $M-T_{\bar{\nu}_{e}}$ plane. Thus statements about the most likely value of $a_{2}$ in this smaller- $a_{2}$ regime will be sensitive to the ill-constrained information on these neutron-star parameters. Consequently we will not make any such statements here. However, the two rightmost panels give us confidence in our ability to derive a bound on $a_{2}$ - as opposed to a most-likely value - since it is clear that certain values of this coupling can be well-excluded, completely independent of details of the PNS modeling.

With the likelihood function already in hand, the derivation of such a bound is quite a simple matter. A natural ansatz for the probability of $a_{n}$ to be smaller than some specified $a_{n}^{0}$ is 

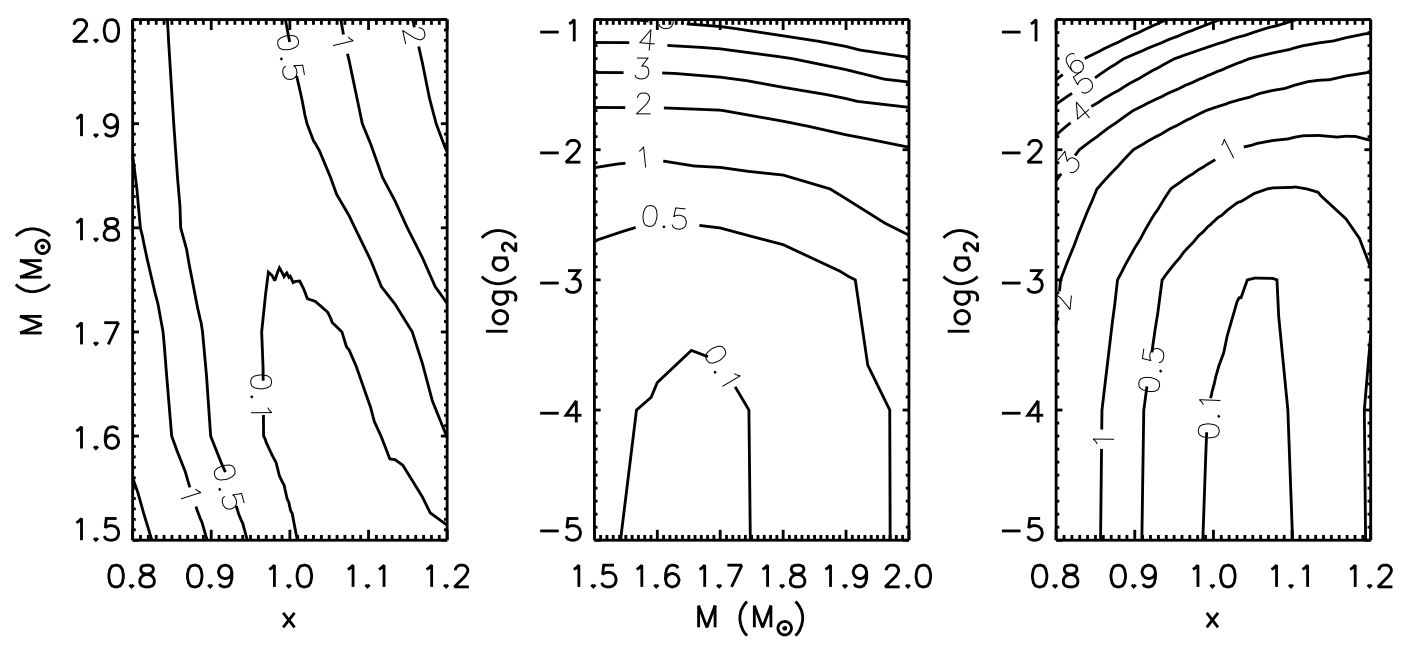

Fig. 2. Contour levels of the function $q$ defined in Eq. (6): $(x-M)$ left panel; $\left(M-\log \left(a_{2}\right)\right)$ middle panel; $\left(x-\log \left(a_{2}\right)\right)$ right panel.

$$
\begin{aligned}
\operatorname{prob}\left(a_{n} \leq a_{n}^{0} \mid\{d a t a\}, I\right)=N \int_{0}^{a_{n}^{0}} d a \int_{0}^{\infty} & d M g(M) \int_{0}^{\infty} d T_{\bar{\nu}_{e}} f\left(T_{\bar{\nu}_{e}}, M\right) \\
& \times \mathcal{L}\left(\{\text { data }\} \mid a_{n}, M, T_{\bar{\nu}_{e}}, I\right) .
\end{aligned}
$$

In other words, we now integrate (or "marginalize") over all possible values of $M$ and $T_{\bar{\nu}_{e}}$, using appropriate weight functions. These functions are $f\left(T_{\bar{\nu}_{e}}, M\right)$, which is the probability for the neutrinosphere to be at a temperature $T_{\bar{\nu}_{e}}$ given a PNS mass, $M$, and $g(M)$, which is the probability for that PNS mass to occur. The overall constant $N$ is defined by the normalization condition $\operatorname{prob}\left(a_{n} \geq 0 \mid\{\right.$ data $\left.\}, I\right)=1$.

A rigorous derivation of this expression can be given by employing Bayesian statistics [28]. In the course of this derivation the weight functions $f$ and $g$ acquire the aforementioned probabilistic interpretations. Note that the structure of Eq. (7) is easily generalized: for every parameter not well determined from other sources one must sum over the parameter space with the appropriate relative weight. In our case we will assume that the mass of the PNS lies within a certain range $\left[M_{\min }, M_{\max }\right]$, but that within that range all values are equally probable. i.e., we write $g(M) \propto \theta\left(M-M_{\min }\right) \theta\left(M_{\max }-M\right)$. Meanwhile, the central value of the anti-neutrino temperature is calculated according to the optical-depth prescription, and $T_{\bar{\nu}_{e}}$ is then assumed o be Gaussian distributed with a $10 \%$ width, independent of time. It might be argued that a potential systematic error exists in the choice of anti-neutrino temperature, and thus a different weight function should be used. To check this we also used a function $f$ which was constant over a range of temperatures that differed by $\pm 20 \%$ from the central value. This altered the bound obtained by less than $10 \%$. 


\section{Improved bounds and discussion}

Equation (7) may now be used to find bounds on $a_{2}$ and $a_{3}$. Defining the bound at the $95 \%$ confidence level to be that value of $a_{n}^{0}$ which obeys $\operatorname{prob}\left(a_{n} \leq\right.$ $a_{n}^{0} \mid\{$ data $\left.\}, I\right)=0.95$, we find that, for the $n=2$ case

$$
a_{2}^{0}=0.022 \mathrm{MeV} / \text { baryon } / \mathrm{s} \text { and thus } R_{2} \leq 0.66 \mu \mathrm{m}
$$

A similar analysis for the $n=3$ case differs in its details, but yields the same overall picture. The bound (again, at the $95 \%$ confidence level) is:

$$
a_{3}^{0}=0.0077 \mathrm{MeV} / \text { baryon } / \mathrm{s} \text { and thus } R_{3} \leq 8 \times 10^{-4} \mu \mathrm{m}
$$

These are within $10 \%$ of the bounds derived in Ref. [4], where the emissivity (1) was derived and the Raffelt criterion applied using $n_{B}=n_{0}$ and a fiducial temperature chosen rather arbitrarily to be $30 \mathrm{MeV}$. The good agreement between this work and Ref. [4] might be thought to suggest that simple criteria based on energetics are an entirely satisfactory. However, analyses of our simulations suggest that the actual fiducial temperature in the $a_{2}=0$ case was $35-40 \mathrm{MeV}$, and the density was as large as $4 n_{0}$ for the most massive stars. Application of the same criterion at these temperatures and densities could have resulted in a bound on $a_{2}$ that was too stringent by a factor of ten. In contrast, the fiducial temperature in our simulations with $a_{2}>0.01$ was generically less than $20 \mathrm{MeV}$, which leads to a bound on $a_{2}$ that is too weak by an order of magnitude. The advantage of our analysis of the SN 1987a data is that it obviates any need to make a subjective judgment about the value of the "real" fiducial temperature. Such an analysis confirms that, for $n=2$ and $n=3$, the supernova bound on the size of extra dimensions found from the neutrino signal of SN 1987a is significantly more stringent than any collider bound which will be obtained in the foreseeable future. It is also, roughly, a factor of hundred more stringent than the bound derived by means of recent tests of sub millimeter gravity [29] for the case of two extra dimensions. We regard this astrophysical information as a robust bound, since our 95\% confidence level is fairly insensitive to uncertainties in the neutron-star mass and the anti-neutrino temperature $\square$. These bounds are specific to scenarios where the extra dimensions are flat or weakly warped [32]. They come about because of the existence of a large number of low-lying KK modes with energy less the characteristic temperature of the supernova core, and thus do not apply to extra-dimensional models where such low-lying KK modes are absent [33].

\footnotetext{
7 Arguments from cosmology [30,31] may provide tighter constraints on $R_{2}$ and $R_{3}$, but there is much we presently do not understand about cosmology in the presence of GODs.
} 
We have not studied the extent to which changes in the neutrino mean-free path or the equation of state of strongly-interacting matter at high density influence our results. Recent work on the influence of many-body correlations on the neutrino-transport mean-free path indicates that the mean-field value for this quantity may be a factor of two or three too small [34]. However, as discussed in Ref. [20] in the axion-radiation case, increases in the meanfree path actually strengthen the bound on exotic-particle couplings, since the shorter neutrino diffusion times that result make it harder to reconcile the late-time neutrino signal with the presence of exotic radiation mechanisms. Consequently we view the bound derived here as one that is conservative in regard to its assumptions about neutrino mean-free paths.

A potentially more significant physics uncertainty is our lack of knowledge about the specific heat of matter at high density. Changes in this function might lead to a large effect on the bounds for $R_{2}$ and $R_{3}$, since they will produce different interior temperatures for the PNS, and the KK-graviton emissivity is a strong function of temperature. However, any changes in the PNS's interior temperature will also affect the neutrino diffusion time since neutrino mean-free paths are strongly temperature dependent. More information on the neutrino spectrum, neutron-star mass and interior physics of the PNS will undoubtedly shed further light on the problem. However, the likelihood function decreases rapidly as $a_{2}$ and $a_{3}$ are increased (see Fig. 2 for $a_{2} \geq 0.01$ ), and so the bounds on these quantities may not be greatly affected by advances in our knowledge of the physics which governs the propagation of energy in the PNS interior.

In contrast, the bound derived here is sensitive to the rate at which energy is radiated to the GODs, i.e. to the temperature-dependence of the graviton emissivity. In particular, many-body effects, such as the multiple-scattering suppression of bremsstrahlung reactions which occurs in a dense plasma and is known as the Landau-Pomeranchuk-Migdal effect, might strongly reduce the KK-gravistrahlung energy-loss rate. Indeed, earlier studies [20] of the multiplescattering suppression of axion radiation due to strong nucleon-spin fluctuations showed that the axion-mass bound was weakened by a factor of two when such effects were considered. Many-body effects might reasonably be expected to have a similar influence here - although gravitons couple to the stress-energy tensor, and not, as axions do, to the nucleon spin, and so they are sensitive to different correlations in the dense plasma. All of these issues warrant further investigation and they will be addressed in future work. In this work we have derived the bounds (8) and (9), which improve upon previous SN 1987a bounds on the size and scales of GODs in three important ways:

(1) They represent the first self-consistent simulations of the early, neutrinoemitting phase of a proto-neutron star which also include energy losses due to the coupling of the Kaluza-Klein modes of the graviton have been 
employed to examine the SN 1987a neutrino signal in detail.

(2) They use the KK-graviton emissivities of Ref. [4], thereby anchoring the nuclear physics of the emissivity for the $N N$ KK-gravistrahlung on solid ground.

(3) They are derived using a rigorous probabilistic analysis that facilitates an unambiguous criterion for the likelihood of certain values of the GODs' radii, and a consequent estimation of confidence levels.

While these improvements do not alter the earlier estimated bounds significantly they do serve to place them on a firm theoretical basis. We also believe that in the event of a future galactic supernova the formalism and ideas developed in this work will prove useful in analyses of the large numbers of counts expected in current-generation neutrino detectors, such as Super Kamiokande and SNO.

Acknowledgements: We are grateful to M. J. Savage, W. Haxton, J. Lattimer and M. Prakash for useful discussions. J. A. P. and D. R. P. are grateful to the Institute for Nuclear Theory and to Iraj Afnan of Flinders University for their hospitality at various stages of this work. We thank the U. S. Department of Energy for its support under DOE grants FG03-97ER4014, FG06-00ER41132 and FG02-87ER40317. C. H. acknowledges the support of the Alexander von Humboldt foundation.

\section{References}

[1] N. Arkani-Hamed, S. Dimopoulos and G. Dvali, Phys. Lett. B429, 263 (1998) hep-ph/9803315.

[2] N. Arkani-Hamed, S. Dimopoulos and G. Dvali, Phys. Rev. D 59, 086004 (1999) hep-ph/9807344.

[3] S. Cullen and M. Perelstein, Phys. Rev. Lett. 83, 268 (1999) hep-ph/9903422.

[4] C. Hanhart, D. R. Phillips, S. Reddy and M. J. Savage, nucl-th/0007016.

[5] G.G. Raffelt, in Stars as Laboratories for Fundamental Physics, University of Chicago Press, Chicago (1996).

[6] K. Hirata et al. [KAMIOKANDE-II Collaboration], Phys. Rev. Lett. 58, 1490 (1987).

[7] R. M. Bionta et al., Phys. Rev. Lett. 58, 1494 (1987).

[8] A. Burrows and J. M. Lattimer, Astrophys. J. 307, 178 (1986).

[9] A. Burrows, Astrophys. J. 334, 891 (1988).

[10] W. Keil and H. T. Janka, Astron. Astrophys. 296, 145 (1995). 
[11] J. A. Pons, S. Reddy, M. Prakash, J. M. Lattimer and J. A. Miralles, Astrophys. J. 513, 780 (1999) astro-ph/9807040.

[12] J. A. Pons, J. A. Miralles, M. Prakash and J. M. Lattimer, Astrophys. J., in press (2001) astro-ph/0008389.

[13] I. Goldman, Y. Aharonov, G. Alexander and S. Nussinov, Phys. Rev. Lett. 60, 1789 (1988).

[14] G. Raffelt and D. Seckel, Phys. Rev. Lett. 60, 1793 (1988).

[15] M. S. Turner, Phys. Rev. Lett. 60, 1797 (1988).

[16] J. M. Lattimer and J. Cooperstein, Phys. Rev. Lett. 61, 23 (1988).

[17] R. Barbieri and R. N. Mohapatra, Phys. Rev. Lett. 61, 27 (1988).

[18] A. Burrows, M. S. Turner and R. P. Brinkmann, Phys. Rev. D 39, 1020 (1989).

[19] E. W. Kolb, R. N. Mohapatra and V. L. Teplitz, Phys. Rev. Lett. 77, 3066 (1996) hep-ph/9605350.

[20] W. Keil, H. Janka, D. N. Schramm, G. Sigl, M. S. Turner and J. Ellis, Phys. Rev. D 56, 2419 (1997) astro-ph/9612222.

[21] B. Jegerlehner, F. Neubig and G. Raffelt, Phys. Rev. D 54, 1194 (1996) astro$\mathrm{ph} / 9601111$.

[22] H. T. Janka, astro-ph/0008432.

[23] M. Rampp and H. T. Janka, Astrophys. J. 539, L33 (2000) astro-ph/0005438.

[24] S. Reddy, M. Prakash and J. M. Lattimer, Phys. Rev. D 58, 013009 (1998) astro-ph/9710115.

[25] D.N. Schramm, L.E. Brown, in Supernova, Vol 6, eds. J.C. Wheeler, T. Piran, S. Weinberg (World Scientific Publishing Co., Singapore), 261 (1990).

[26] G. G. Raffelt, Phys. Rept. 198, 1 (1990).

[27] T.J. Loredo, D.Q. Lamb, in Proceedings of the $14^{\text {th }}$ Texas Symposium on Relativistic Astrophysics, Ann. N.Y. Acad. Sci. 571 (1989) 601.

[28] For a very pedagogical introduction see D. S. Sivia, Data Analysis/ A Bayesian Tutorial, Oxford University Press Inc., New York, (1996).

[29] C. D. Hoyle, U. Schmidt, B. R. Heckel, E. G. Adelberger, J. H. Gundlach, D. J. Kapner and H. E. Swanson, hep-ph/0011014.

[30] L. J. Hall and D. Smith, Phys. Rev. D 60, 085008 (1999) hep-ph/9904267.

[31] M. Fairbairn, hep-ph/0101131.

[32] P. J. Fox, hep-ph/0012165.

[33] L. Randall and R. Sundrum, Phys. Rev. Lett. 83, 3370 (1999) hep-ph/9905221.

[34] S. Reddy, M. Prakash, J. M. Lattimer and J. A. Pons, Phys. Rev. C 59, 2888 (1999) astro-ph/9811294. 